OESOPHAGUS

\title{
Pneumatic dilation for achalasia: late results of a prospective follow up investigation
}

\author{
V F Eckardt, I Gockel, G Bernhard
}

Gut 2004;53:629-633. doi: 10.1136/gut.2003.029298

See end of article for authors' affiliations ....................

Correspondence to: Professor Dr V F Eckardt, Deutsche Klinik für Diagnostik, Aukammallee 33, 65191 Wiesbaden, Germany; eckardt. gastro@dkd-wiesbaden.de

Accepted for publication 12 November 2003

\begin{abstract}
Background and aims: In this prospective study, we determined the long term clinical course of patients with achalasia who were treated by pneumatic dilation using the Browne-McHardy dilator, and determined whether previously described predictors of outcome remain significant after prolonged follow up.

Methods: Between 1981 and 1991, 54 consecutive patients were treated by pneumatic dilation and followed up at regular intervals for a median of 13.8 years. Remission was determined with the use of a structured interview and a previously described symptom score. Duration of remission was evaluated by Kaplan-Meier estimates of time to recurrence. Predictors of outcome were determined using the log rank test.

Results: Complete follow up until 2002 was obtained in $98 \%$ of all patients. Seven patients had died and were censored. A single pneumatic dilation resulted in a five year remission rate of $40 \%$ and a 10 year remission rate of $36 \%$. Repeated dilations only mildly improved the clinical response. Patients who were older than 40 years had a significantly better outcome than younger patients (log rank test, $p=0.0014$ ). However, the most significant predictive factor for a favourable long term outcome was a post-dilation lower oesophageal sphincter pressure of less than $10 \mathrm{~mm} \mathrm{Hg}$ (log rank test, $p=0.0001$ ).

Conclusions: Long term results of pneumatic dilation are less favourable than previously thought. Young patients and those not responding to a single pneumatic dilation should be offered alternative therapy. Patients who remain in remission for five years are likely to benefit from the longlasting treatment effect of pneumatic dilation.
\end{abstract}

$\mathrm{P}$ atients with achalasia may be effectively treated by either surgical myotomy or pneumatic dilation. ${ }^{12}$ While it remains unknown which of these treatment modalities leads to the most optimal long term response, most gastroenterologists prefer pneumatic dilation as firstline therapy. However, the effectiveness of the latter therapy has been investigated mainly in retrospective studies and there is little information with regard to the patient's long term clinical course following a single pneumatic dilation or after repeated therapies.

In 1992, we reported a prospective observation of 54 patients who had been treated with pneumatic dilation and were followed for a median of 3.8 years. ${ }^{3}$. Since then, the same patient cohort were regularly reassessed at two year intervals until the summer of 2002, thereby prolonging the observation period to a maximum of 19 years and obtaining a median follow up time of 13.8 years. The aim of this reassessment was twofold: (1) to determine whether patients who had reached clinical remission after a single or multiple dilations remained "asymptomatic" for a prolonged period of time; and (2) to re-evaluate whether factors suggesting a favourable response (age over 40 years and post-dilation lower oesophageal sphincter pressure of less than $10 \mathrm{~mm} \mathrm{Hg}$ ) retained their predictive value over a prolonged observation period.

\section{MATERIALS AND METHODS \\ Patients}

From 1981 to 1991, 54 consecutive and newly diagnosed patients with achalasia underwent pneumatic dilation by the senior investigator (VFE). In each instance, the diagnosis of achalasia was based on the results of radiographic, endoscopic, and manometric studies. Patients comprised 33 males and 21 females, and ranged in age from 13 to 86 years (mean age 42.7 (SD 17.7) years). With the exception of a single patient who was lost to follow up after five years of observation, all patients (98\%) were followed until the final interview in 2002 or until death.

\section{Evaluation of symptoms}

At the initial investigation, four weeks later, and every two years thereafter, structured interviews were performed using a previously described symptom score. ${ }^{4}$ Depending on whether dysphagia, regurgitation, and chest pain occurred occasionally, daily, or several times during the day, a symptom score between 0 and 3 was determined. In addition, a symptom score of $0-3$ was assigned to the degree of weight loss. Thus a completely asymptomatic patient would have a symptom score of 0 whereas a severely affected patient could have a symptom score of up to 12. Patients were considered to have reached clinical remission if symptoms had totally disappeared or if they had improved by at least two points and did not exceed a score of 3. Patients who requested further therapy despite having a symptom score of less than 4 were also considered treatment failures.

\section{Pneumatic dilation}

Pneumatic dilation was carried out with the use of a BrowneMcHardy dilator under fluoroscopic control. Only the youngest patient ( 13 years old) received premedication with diazepam; the remaining patients received topical anaesthesia of the pharynx only. The balloon of the dilator was placed fluoroscopically at the gastro-oesophageal junction and inflated until a minimum pressure of 6 pSI was reached and the waist remained in a stable position. Depending on the patient's tolerance, pressures ranged from 6 to $12 \mathrm{pSI}$

Abbreviations: LOS, lower oesophageal sphincter 


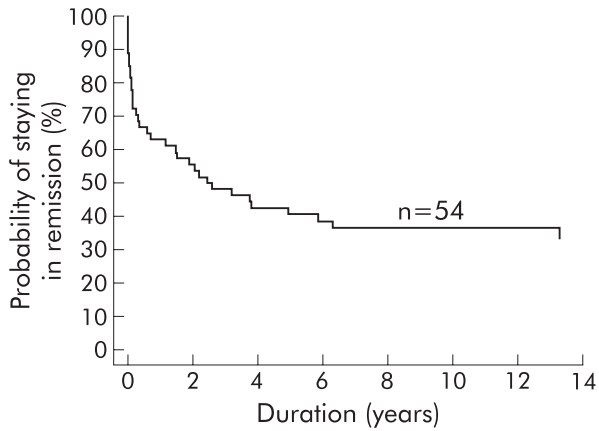

Figure 1 Kaplan-Meier curve. Time to become symptomatic after the first dilatation.

with the aim of maintaining this pressure for approximately two minutes and to obliterate the waist of the balloon. Nine patients developed significant pain and the procedure had to be terminated prematurely (after 30-60 seconds). Whenever possible, a radiograph of the oesophagogastric junction was obtained when the waist of the balloon (that is, its midportion) had reached its maximum diameter.

\section{Post-dilation investigations}

Prior to pneumatic dilation and four weeks thereafter, patients underwent manometric studies with the use of a low compliance capillary perfusion method and a catheter assembly, with three side holes which were situated $5 \mathrm{~cm}$ apart and were radially oriented at $120^{\circ}$ angles. ${ }^{3}$ During this procedure, the lower oesophageal sphincter (LOS) pressure was determined by a station pull through method. The mean of two complete pull throughs, representing six individual pressure measurements, was determined as the LOS resting pressure. In eight patients, during the initial examination, the tube assembly could not be passed into the stomach. However, oesophageal body motility occurring in response to 10 wet swallows was recorded in all patients and always revealed the absence of peristalsis. In seven of these eight patients, LOS pressure determinations became possible following pneumatic dilation and always revealed incomplete sphincter relaxation.

Following pneumatic dilation, all patients or their relatives and physicians were contacted and interviewed at two yearly intervals. In case of death, further information was obtained from physician records and hospital charts. Follow up was continued until the summer of 2002, 11 years after the last patient had been enrolled.

\section{Statistical analysis}

Patient characteristics, symptoms, as well as the results of manometric measurements are described using summary statistics. Quantitative variables are summarised using counts (n), means, standard deviations (SD), medians, and ranges (that is, minimum and maximum values). Qualitative or categorical variables are described using frequencies (n) and percentages $(\%)$.

Statistical analysis focused on determining the recurrence free survival for the entire patient group, as well as subgroups, using Kaplan-Meier estimates. ${ }^{5}$ The probability of staying at least five years in remission is presented as a five year remission rate with $95 \%$ confidence interval (95\% CI) (lower limit 95\% CI, upper limit 95\% CI). ${ }^{6}$

The associations between baseline and post-baseline factors and recurrence free survival were investigated using the Kaplan-Meier estimate of time to event and were compared using log rank test statistics. Baseline factors considered in this analysis were age and post-dilation LOS pressure, both of which were previously shown to have a significant impact on patient outcome following pneumatic dilation. ${ }^{3}$ The predictive value of age at diagnosis and postdilation LOS pressures was estimated with the use of a Cox proportional hazards model. The assumed linear regression effects of age and post-dilation LOS pressures were checked using Martingale residuals and were found to be satisfactory. The probability of remaining in remission and 95\% CI were calculated for two and 10 years in relation to these parameters.

\section{RESULTS}

\section{Overall outcome}

During a mean follow up of 13.6 (3.4) years (range 1.6-18.6), 35 of 54 patients experienced clinical relapse. Five years following a single pneumatic dilation, $60 \%$ of all patients had again become symptomatic (fig 1). Thereafter, only a few recurrences were observed. Of the 21 patients who were still in remission at five years, only three (14\%) experienced a relapse within the following 10 years.

As shown in table 1, during the observation period, seven patients died and were censored. In one, death was due to oesophageal squamous cell cancer, indicating an oesophageal cancer incidence of 1 in 734 patient years. This patient had symptoms of achalasia since 1978 and was successfully treated with a single pneumatic dilation in 1987. She had remained asymptomatic until 1998 when she was diagnosed as having squamous cell cancer of the mid-oesophagus. Two years later she died of disseminated cancer. The patient who died of pneumonia was treated with pneumatic dilation in 1988 and did not report oesophageal symptoms until her death in 2001. Since 1999 she had been treated in a nursing home for Alzheimer's disease. There was no history of aspiration prior to her final illness.

\section{Immediate and late complications of pneumatic dilation}

A single patient experienced a perforation which was recognised five hours following dilation therapy. She was treated conservatively and had an uneventful hospital course. Two further patients developed intramural haematomas that

Table 1 Characteristics of the deceased patients

\begin{tabular}{|c|c|c|c|}
\hline Sex & $\begin{array}{l}\text { Age at diagnosis of } \\
\text { achalasia (y) }\end{array}$ & $\begin{array}{l}\text { Age at death } \\
\text { (y) }\end{array}$ & Cause of death \\
\hline Male & 63 & 75 & Cancer of urinary bladder \\
\hline Male & 21 & 24 & Leukaemia \\
\hline Male & 80 & 91 & Cardiovascular disease \\
\hline Female & 74 & 87 & Pneumonia \\
\hline Female & 75 & 85 & Adhesion ileus \\
\hline Female & 86 & 88 & Stroke \\
\hline Female & 43 & 56 & Oesophageal squamous cell cancer \\
\hline
\end{tabular}




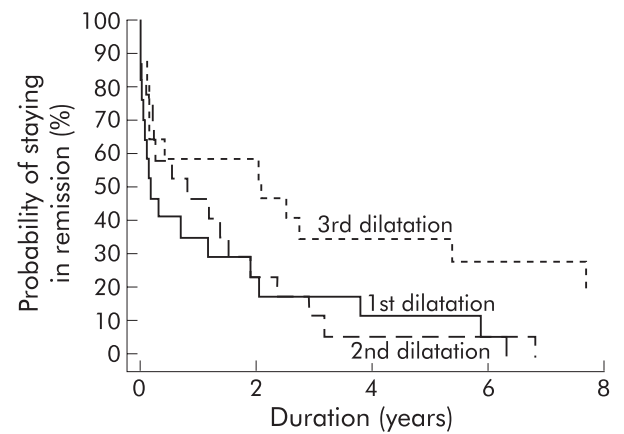

Figure 2 Kaplan-Meier curve. Time to become symptomatic after the first, second, and third dilatation for patients receiving three dilatations.

resolved spontaneously within 48 hours. Six patients developed reflux oesophagitis during further follow up, all of whom became asymptomatic with medical therapy.

\section{Effect of repeated dilation on outcome}

Patients undergoing more than one dilation had a mildly better outcome following the second dilation. For these patients, the five year remission rate was 6\% (95\% CI $0 \%$, $15 \%)$ after the first and $28 \%(13 \%, 44 \%)$ after the second dilation. Patients requiring three dilations did not have a better long term response after the second compared with the first dilation (fig 2$)$. At five years, only $12 \%(0 \%, 27 \%)$ and $6 \%$ $(0 \%, 17 \%)$ remained in clinical remission following the first and second dilation. A third dilation improved the clinical remission rate to $35 \%(13 \%, 58 \%)$ at five years but to only $19 \%(0 \%, 40 \%)$ at 10 years.

\section{Effect of age on long term outcome}

As shown in fig 3 , young patients $(\leqslant 40$ years) responded poorly to a single pneumatic dilation. Their calculated five year remission rate was only $16 \%$ (95\% CI 1\%, 32\%). In contrast, $58 \%(40 \%, 76 \%)$ of patients who were older than 40 years remained in clinical remission at five years and $52 \%$ $(33 \%, 69 \%)$ were still free of symptoms at 10 years. The difference between the two groups was confirmed using the $\log$ rank test statistics $(\mathrm{p}=0.0014, \mathrm{df}=1)$. Patients who were younger than 20 years $(n=6)$ had the poorest response. Only one remained in remission for more than two years. Based on these data, the probability of remission at two and 10 years was estimated for different ages at first diagnosis using a Cox proportional hazards model. As illustrated in fig 4, a patient who first presents with achalasia at the age of 30 years has a $28 \%(16 \%, 48 \%)$ probability of remaining in remission for 10 years, while a 70 year old patient has a probability of $53 \%$ $(34 \%, 82 \%)$.

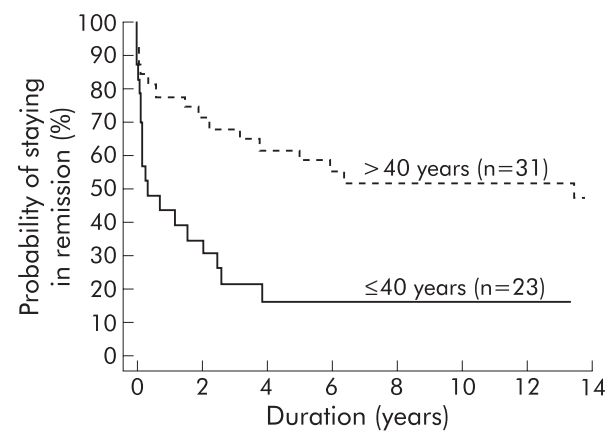

Figure 3 Kaplan-Meier curve. Time to become symptomatic by patient age (up to 40 years and older than 40 years).

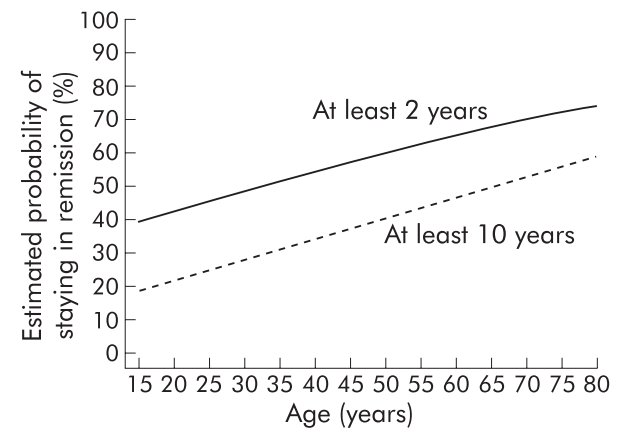

Figure 4 Estimated probability of staying in remission (from the Cox proportional hazards model) after the first dilatation for at least two years and at least 10 years by age.

Post-dilation LOS pressure as a predictor of outcome Patients who achieved a post-dilation LOS pressure of less than $10 \mathrm{~mm} \mathrm{Hg}$ had an excellent long term clinical response (fig 5). Seventy five per cent (95\% CI 60\%, 90\%) were still in remission after 10 years. In contrast, the 10 year remission rate decreased to $53 \%(32 \%, 75 \%)$ for those with a postdilation LOS pressure of $10-20 \mathrm{~mm} \mathrm{Hg}$ and to $13 \%(0 \%, 29 \%)$ for patients with even higher pressures. The two patients with post-dilation sphincter pressures of $<10 \mathrm{~mm} \mathrm{Hg}$ who experienced a late relapse had redeveloped sphincter hypertension (18.3 and $23.3 \mathrm{~mm} \mathrm{Hg}$ ) when symptoms recurred.

Post-dilation LOS pressure highly predicted clinical outcome after pneumatic dilation, the difference between the three groups was again confirmed by the log rank test statistics $(p=0.0001, d f=2)$. On the basis of these values, the probability of remission was calculated for different postdilation sphincter pressures with the use of a Cox proportional hazards model. Such calculations shows (fig 6) for example that a patient with a post-dilation LOS pressure of $10 \mathrm{~mm} \mathrm{Hg}$ will have a $66 \%$ (95\% CI 52\%, 85\%) chance of remaining in remission for 10 years while this probability drops to $31 \%(19 \%, 50 \%)$ if post-dilation LOS pressure is $20 \mathrm{~mm} \mathrm{Hg}$.

\section{DISCUSSION}

This study shows that the long term clinical response to pneumatic dilation is less favourable than previously thought. Young patients and those who do not achieve a significant decrease in LOS pressure after a single pneumatic dilation are unlikely to achieve long term clinical remission. However, patients who remain in clinical remission at five years have an excellent chance of requiring no further therapy for prolonged periods of time.

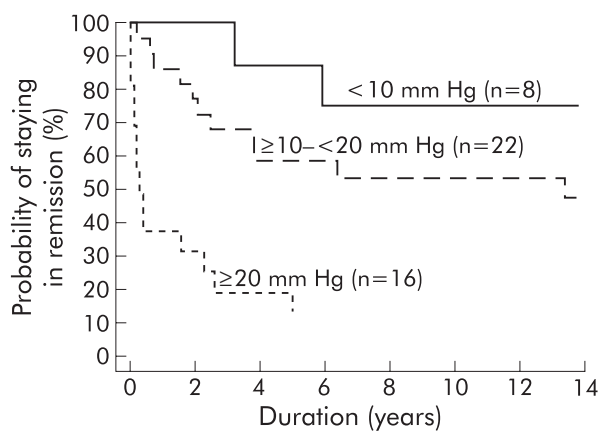

Figure 5 Kaplan-Meier curve. Time to become symptomatic by patient post-dilatation lower oesophageal sphincter pressure $(<10 \mathrm{~mm} \mathrm{Hg}$, $10-<20 \mathrm{~mm} \mathrm{Hg}$, $\geqslant 20 \mathrm{~mm} \mathrm{Hg}$ ). 


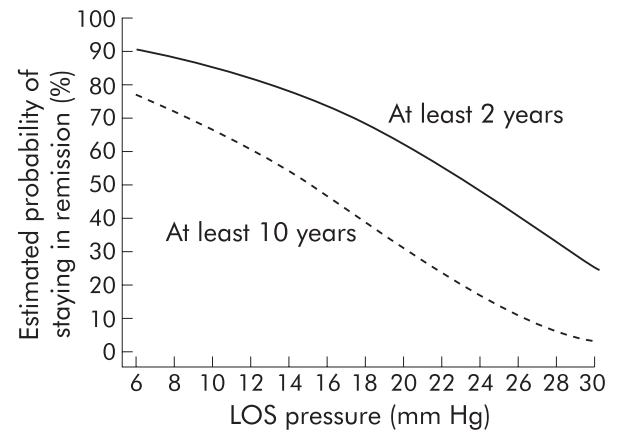

Figure 6 Estimated probability of staying in remission (from the Cox proportional hazards model) after the first dilatation for at least two years and at least 10 years by post-dilatation lower oesophageal sphincter (LOS) pressure.

To our knowledge, to date a total of 19 prospective investigations including more than 10 patients have evaluated the efficacy of pneumatic dilation in patients with achalasia. $^{3-24}$ In these studies, the overall excellent or good symptomatic response was reported to range from $61 \%$ to $100 \%$. However, interpretation of such data is hampered by the fact that most authors used vague criteria to define a clinical response and that the mean duration of follow up never exceeded five years. The current prospective investigation overcomes some of these methodological shortcomings in that stringent criteria for clinical remission were used and regular follow up extended for a mean period of almost 14 years. During this prolonged observation period, only $40 \%$ of patients treated with a single pneumatic dilation remained in remission at five years. Thereafter, recurrences became an exception. The results are almost identical to those reported recently in a retrospective investigation. ${ }^{25}$ Only $40 \%$ of patients followed for 15 years or longer were considered to have an excellent or good clinical response. However, one caveat to these observations should be applied. In both studies either compliant or "home made" dilators were used, none of which is commonly used today. It is therefore difficult to extrapolate the findings of our study to current clinical practice. In fact, some have suggested that the newer dilators, such as the non-compliant Rigiflex dilator, are slightly more effective than the older type dilators. ${ }^{26}$ However, the only randomised comparison of compliant versus noncompliant dilators did not detect such differences. ${ }^{9}$ Thus it remains to be determined whether better long term results of pneumatic dilation can be obtained with the newer dilators.

The question must be raised whether the results of a single pneumatic dilation can be improved by repeated therapies. In our previously reported short term observational study, ${ }^{3}$ we concluded that additional treatments not only repeat the initial success but are followed by a progressively longer duration of remission. These conclusions must now be modified. Although there was still a tendency for further improvement after the second and third dilation, both therapies resulted in a less than $30 \%$ chance of remaining in remission for more than five years. Thus we believe that failure to respond to a single pneumatic dilation, performed under optimal conditions, should lead to consideration of an alternative therapy such as laparoscopic myotomy.

In the hope of optimising treatment of achalasia and to tailor such therapy to the individual patient, several authors have tried to determine factors that predict clinical outcome following pneumatic dilation. In these studies, advanced age, a moderately dilated oesophagus, and a long history of symptoms prior to diagnosis were all found to predict a favourable treatment result. ${ }^{37-33}$ However, the most significant factor predicting treatment response appears to be age at diagnosis. The results of the current study confirm and strengthen our previous observation that patients who are younger than 40 years poorly respond to pneumatic dilation. Following a single treatment session, less than $20 \%$ of these younger patients still remained in clinical remission after five years, while patients older than 40 years had a five year remission rate of $58 \%$. The reason for these differences in treatment response remains unknown. However, it has been speculated that a general difference in muscle strength across the age groups may account for this observation. ${ }^{34}$

Another approach in predicting a patient's long term clinical outcome following pneumatic dilation is to perform objective evaluation of oesophageal function four weeks after therapy. For this purpose, oesophageal scintigraphy, barium oesophagogram, and LOS pressure measurements have been used. With regard to scintigraphy, post-therapy studies have only shown correlations with short term symptom improvement while they were unable to predict a patient's long term response. ${ }^{35}$ Most recently, it has been shown that the timed barium swallow ${ }^{36}$ reliably predicts long term remission rates. However, to date, these conclusions are based on observations made in a single centre and on a rather small patient group. The most extensive experience with objective evaluations of treatment responses after pneumatic dilation relates to determination of LOS pressures. ${ }^{3} 14151819$ 37-39 In six of these eight investigations, symptom improvement correlated significantly with a decrease in LOS pressure and the most optimal response was found in patients exhibiting a postdilation sphincter pressure of less than $10 \mathrm{~mm} \mathrm{Hg} .{ }^{314}$ The current study extends these observations by showing that post-dilation LOS pressure retains its ability to predict the long term clinical response even if patients are followed for more than 10 years. In fact, a post- dilation pressure below $10 \mathrm{~mm} \mathrm{Hg}$ remains the most favourable and significant prognostic variable. Of eight patients reaching this goal following a single pneumatic dilation, only two became symptomatic after three and six years. Thus the great majority of patients for whom pneumatic dilation diminishes LOS pressure to levels below $10 \mathrm{~mm} \mathrm{Hg}$ will remain asymptomatic for many years.

In summary, we conclude from the results of this prospective long term observation that young patients with achalasia respond poorly to pneumatic dilation. Whether these patients benefit more from immediate surgery remains to be shown in a prospective controlled trial. Similarly, repeated dilations rarely result in long term clinical remission. The question must therefore be raised whether failure to respond to a first dilation should lead to consideration of alternative therapy.

\section{Authors' affiliations \\ V F Eckardt, I Gockel, Deutsche Klinik für Diagnostik, Wiesbaden, Germany \\ G Bernhard, Department of Biostatistics, Novartis Pharma AG, Basel, Switzerland}

\section{REFERENCES}

1 Kadakia SC, Wong RKH. Pneumatic balloon dilation for esophageal achalasia. Gastrointest Endosc Clin N Am 2001;11:325-45.

2 Ali A, Pellegrini CA. Laparoscopic myotomy. Technique and efficacy in treating achalasia. Gastrointest Endosc Clin N Am 2001;11:347-57.

3 Eckardt VF, Aignherr C, Bernhard G. Predictors of outcome in patients with achalasia treated by pneumatic dilation. Gastroenterology 1992;103:1732-8.

4 Eckardt VF. Clinical presentation and complications of achalasia. Gastrointest Endosc Clin N Am 2001;11:281-92.

5 Kaplan EL, Meier P. Nonparametric estimation from incomplete observations. J Am Stat Assoc 1958;53:457-81. 
6 Gardner MJ, Altman DG. Estimating with confidence. BMJ (Clin Res Ed) 1988;296:1210-11.

7 Heimlich HJ, O'Connor TW, Flores DC. Case for pneumatic dilatation in achalasia. Ann Otol Rhinol Laryngol 1978;87:519-22.

8 Csendes A, Braghetto I, Henriquez A, et al. Late results of a prospective randomised study comparing forceful dilatation and oesophagomyotomy in patients with achalasia. Gut 1989;30:299-304.

9 Stark GA, Castell DO, Richter JE, et al. Prospective randomized comparison of Brown-McHardy and microvasive balloon dilators in treatment of achalasia. Am J Gastroenterol 1990;85:1322-6.

10 Wong RC, Maydanovitch C. Utility of parameters measured during pneumatic dilation as predictors of successful dilation. Am J Gastroenterol 1996;91:1126-9

11 Elta GH, Nostrant TT, Wilson JA. Treatment of achalasia with the Witzel pneumatic dilator. Gastrointest Endosc 1987;33:101-3.

12 Johnston BT, Collins BJ, Collins JS, et al. Perendoscopic pneumatic dilatation in achalasia: assessment of outcome using esophageal scintigraphy. Dysphagia, 19992, 7:201-4

13 Mearin F, Armengol JR, Chicharro L, et al. Forceful dilatation under endoscopic control in the treatment of achalasia: a randomised trial of pneumatic versus metallic dilators. Gut 1994;35:1360-2.

14 Ponce J, Garrigues V, Pertejo V, et al. Individual prediction of response to pneumatic dilation in patients with achalasia. Dig Dis $S_{c i}$ 1996:41:2135-41.

15 Gelfand MD, Kozarek RA. An experience with polyethylene balloons for pneumatic dilation in achalasia. Am J Gastroenterol 1989;84:924-7.

16 Barkin JS, Guelrud M, Reiner DK, et al. Forceful balloon dilation: an outpatient procedure for achalasia. Gastrointest Endosc 1990;36:123-6.

17 Lee JD, Cecil BD, Brown PE, et al. The Cohen test does not predict outcome in achalasia after pneumatic dilation. Gastrointest Endosc 1993;39:157-60.

$18 \mathrm{Kim} \mathrm{CH}$, Cameron AJ, Hsu JJ, et al. Achalasia: prospective evaluation of relationship between lower esophageal sphincter pressure, esophageal transit, and esophageal diameter and symptoms in response to pneumatic dilation. Mayo Clin Proc 1993;68:1067-73.

19 Kadakia SC, Wong RK. Graded pneumatic dilatation using Rigiflex achalasia dilators in patients with primary esophageal achalasia. Am J Gastroenterol 1993;88:34-8.

20 Wehrmann $T$, Jacobi V, Jung $M$, et al. Pneumatic dilation in achalasia with a low-compliance balloon: results of a 5-year prospective evaluation. Gastrointest Endosc 1995;42:31-6.

21 Muehldorfer SM, Hahn EG, Ell C. High- and low-compliance balloon dilators in patients with achalasia: a randomized prospective comparative trial. Gastrointest Endosc 1996;44:398-403.
22 Bhatnagar MS, Nanivadekar SA, Sawant P, et al. Achalasia cardia dilatation using polyethylene balloon (Rigiflex) dilators. Indian J Gastroenterol 1996;15:49-51.

23 Gideon RM, Castell DO, Yarze J. Prospective randomized comparison of pneumatic dilatation technique in patients with idiopathic achalasia. Dig Dis Sci 1999;44:1853-7.

24 Becker K, Biesenbach S, Erckenbrecht JF, et al. Effect of balloon compliance on symptomatic success of pneumatic dilation in achalasia patients. Z Gastroenterol 2001;39:831-6.

25 West RL, Hirsch DP, Bartelsman WM, et al. Long term results of pneumatic dilation in achalasia followed for more than 5 years. Am J Gastroenterol 2002; 97:1346-51.

26 Vaezi MF, Richter JE. Current therapies for achalasia: comparison and efficacy. J Clin Gastroenterol 1998;27:21-53.

27 Parkman HP, Reynolds JC, Ouyang A, et al. Pneumatic dilatation or esophagomyotomy treatment for idiopathic achalasia: Clinical outcomes and cost analysis. Dig Dis Sci 1993;38:75-85.

28 Vantrappen G, Hellemans J. Achalasia. In: Vantrappen G, Hellemans J, eds. Diseases of the esophagus. New York: Springer Verlag, 1974:287-354.

29 Fellows IW, Ogilvie AL, Atkinson M. Pneumatic dilatation in achalasia. Gut 1983;24:1020-3

30 Katz PO, Gilbert J, Castell DO. Pneumatic dilatation is effective long-term treatment for achalasia. Dig Dis Sci 1998:43:1973-7.

31 Chawla YK, Dilawari JB, Reddy DN, et al. Pneumatic dilatation in achalasia cardia. Indian J Gastroenterol 1987;6:101-2.

32 Azizkhan RG, Tapper D, Eraklis A. Achalasia in childhood: a 20-year experience. J Pediatr Surg 1980;15:457-61.

33 Barnett JL, Eisenman R, Nostrant $\Pi$, et al. Witzel pneumatic dilation for achalasia: safety and long-term efficacy. Gastrointest Endosc 1990;36:482-5.

34 Richter JE. Comparison and cost analysis of different treatment strategies in achalasia. Gastrointest Endosc Clin N Am 2001:11:359-69.

35 Vaezi MF. Quantitative methods to determine efficacy of treatment in achalasia. Gastrointest Endosc Clin N Am 2001;11:409-24.

36 Vaezi MF, Baker ME, Achkar E, et al. Timed barium oesophagogram: better predictor of long term success after pneumatic dilation in achalasia than symptom assessment. Gut 2002;50:765-70.

37 Holloway RH, Krosin G, Lange RC, et al. Radionuclide esophageal emptying of a solid meal to quantitate results of therapy in achalasia. Gastroenterology 1983;84:771-6.

38 Shahi HM, Aggarwal R, Misra A, et al. Relationship of manometric findings to symptomatic response after pneumatic dilation in achalasia cardia. Indian J Gastroenterol 1998;17:19-21.

39 Alonso P, Gonzalez-Conde B, Macenlle R, et al. The usefulness of manometry for evaluation of treatment. Dig Dis Sc 1999;44:536-41. 